\title{
Advances in ambient intelligence technologies
}

\author{
Quan Z. Sheng • Elhadi M. Shakshuki • \\ Jiangang Ma
}

Published online: 11 July 2013

(c) Springer-Verlag Berlin Heidelberg 2013

This special issue contains selected papers from the 3rd International Conference on Ambient Systems, Networks and Technologies (ANT 2012), which was held at Niagara Falls, Ontario, Canada, August 27-29, 2012. The conference attracted a large number of scientific papers that contributed to the state-of-the-art in the areas of pervasive and ambient information systems. All the papers selected for this special issue have undergone two rounds of rigorous review process. Based on the reviewers' feedback, seven papers were selected for publication from eight best papers invited from ANT 2012. The accepted papers cover interesting works on new developments in ambient intelligence such as adaptable intelligence systems, smart environments, body area networks, and network security.

The first paper by Zhang, Khedri, and Jaskolka, "An Aspect-Oriented Language for Feature Modeling", proposes a new language called AO-PFA for developing ambient systems. Built on top of Product Family Algebra (PFA) and by using the aspect-oriented technology, AOPFA offers capabilities in dealing with unpredictable changes in ambient applications. The paper by Hoelzl, Kurz, and Ferscha, "Goal Oriented Recognition of Composed Activities for Reliable and Adaptable Intelligence Systems", focuses on activity and context recognition, an

Q. Z. Sheng · J. Ma

School of Computer Science, The University of Adelaide, Adelaide, SA 5005, Australia

e-mail: qsheng@cs.adelaide.edu.au

J. Ma

e-mail: jiangang.ma@adelaide.edu.au

E. M. Shakshuki ( $\square)$

Jodrey School of Computer Science,

Acadia University, Wolfville, Canada

e-mail: elhadi.shakshuki@acadiau.ca important research topic for adaptable ambient intelligence systems. The paper proposes a goal oriented approach to dynamically create and instantiate models to recognize high level composed activities in an opportunistic way.

The paper by $\mathrm{Li}, \mathrm{He}$, and Arora, "ThermoNet: FineGrain Assessment of Building Comfort and Efficiency", describes ThermoNet, a hybrid wireless sensor network solution for monitoring and optimizing energy consumption of the Heating, Ventilation, and Air Conditioning systems in large buildings. The paper reports some interesting findings based on the data collected from a realworld environment. The paper by Menon, Jayaraman, and Govindaraju, "Probabilistic Spatio-Temporal Retrieval in Smart Spaces", deals with uncertainties of object tracking in smart spaces. The paper proposes a data model that supports computation of probabilities of spatio-temporal queries giving the uncertainty of the location of an occupant. The paper by Knapen et al., "Exploiting GraphTheoretic Tools for Matching in Carpooling Applications", studies carpooling for commuting and proposes a Global CarPooling Matching Service (GCPMS) that provides advice on how to combine commuting trips by carpooling. In order to evaluate the matching performance of the GCPMS, a multi-agent system is also developed and some initial experimental results are reported.

The paper by Khan et al. "A New Patient Monitoring Framework and Energy-Aware Peering Routing Protocol (EPR) for Body Area Network Communication", proposes a new architecture of Body Area Networks for indoor hospital environments. The paper presents an EPR for reducing the traffic load and energy consumption while increasing the number of packets successfully delivered to the destinations. The final paper by Beitollahi and Deconinck, "ConnectionScore: A Statistical Technique to Resist Application-Layer DDoS Attacks", describes a 
novel scheme called ConnectionScore against applicationlevel DDoS attacks. ConnectionScore considers historical and statistical information of connections and the analysis shows the effectiveness of the technique in detecting malicious attackers.

The guest editors would like to take this opportunity to thank all the authors for the efforts they put in the preparation of their manuscripts and for their valuable contributions. We wish to express our deepest gratitude to the referees who provided very useful and thoughtful feedback to the authors. Finally, our sincere thanks go to the Editor-in-Chief, Prof. Vincenzo Loia, of Journal of Ambient Intelligence and Humanized Computing for his kind support, advice, and encouragements throughout the preparation of this special issue. 